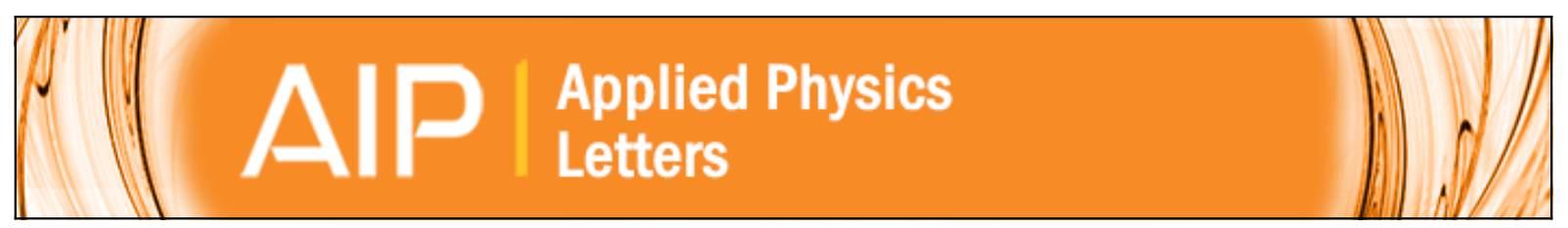

\title{
Bidirectional waveguide coupling with plasmonic Fano nanoantennas
}

Rui Guo, Manuel Decker, Isabelle Staude, Dragomir N. Neshev, and Yuri S. Kivshar

Citation: Applied Physics Letters 105, 053114 (2014); doi: 10.1063/1.4892651

View online: http://dx.doi.org/10.1063/1.4892651

View Table of Contents: http://scitation.aip.org/content/aip/journal/apl/105/5?ver=pdfcov

Published by the AIP Publishing

\section{Articles you may be interested in}

Hybrid nanoantennas for directional emission enhancement

Appl. Phys. Lett. 105, 221109 (2014); 10.1063/1.4903219

Mapping near-field localization in plasmonic optical nanoantennas with $10 \mathrm{~nm}$ spatial resolution

Appl. Phys. Lett. 105, 053105 (2014); 10.1063/1.4892577

Observation of the high-sensitivity plasmonic dipolar antibonding mode of gold nanoantennas in evanescent waves

Appl. Phys. Lett. 105, 031117 (2014); 10.1063/1.4891573

Ultra-sharp plasmonic resonances from monopole optical nanoantenna phased arrays

Appl. Phys. Lett. 104, 231101 (2014); 10.1063/1.4881323

Design of a vertically coupled liquid-crystal long-range plasmonic optical switch

Appl. Phys. Lett. 102, 101103 (2013); 10.1063/1.4794939

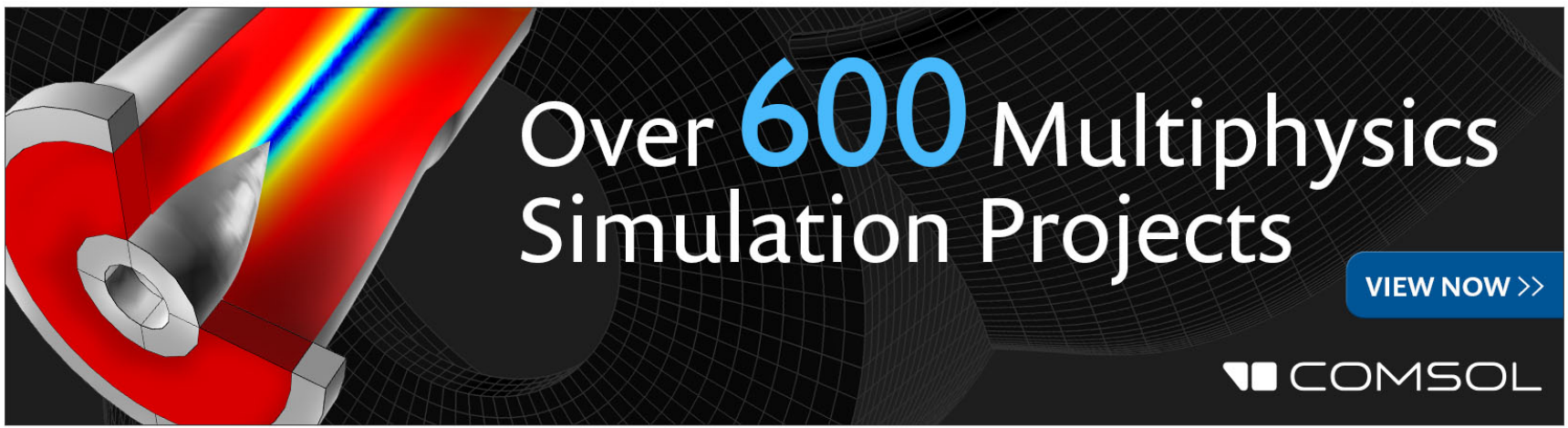




\title{
Bidirectional waveguide coupling with plasmonic Fano nanoantennas
}

\author{
Rui Guo, Manuel Decker, ${ }^{\text {a) }}$ Isabelle Staude, Dragomir N. Neshev, and Yuri S. Kivshar \\ Nonlinear Physics Centre and Centre for Ultrahigh Bandwidth Devices for Optical Systems (CUDOS), \\ Research School of Physics and Engineering, The Australian National University, Canberra ACT 0200, \\ Australia
}

(Received 4 June 2014; accepted 28 July 2014; published online 8 August 2014)

\begin{abstract}
We introduce the concept of a bidirectional, compact single-element Fano nanoantenna that allows for directional coupling of light in opposite directions of a high-index dielectric waveguide for two different operation wavelengths. We utilize a Fano resonance to tailor the radiation phases of a gold nanodisk and a nanoslit that is inscribed into the nanodisk to realize bidirectional scattering. We show that this Fano nanoantenna operates as a bidirectional waveguide coupler at telecommunication wavelengths and, thus, is ideally suitable for integrated wavelength-selective light demultiplexing. (C) 2014 AIP Publishing LLC. [http://dx.doi.org/10.1063/1.4892651]
\end{abstract}

In the last decade, plasmonic nanoparticles and nanoantennas have proven to provide unprecedented opportunities to control and guide light at the nanoscale. ${ }^{1-3}$ Due to their unique ability to confine and enhance light to subwavelength volumes, they provide the basis for the development of novel types of solar cells, photo detectors, imaging devices, advanced plasmonic sensors, and nanolasers. ${ }^{2,4}$ Plasmonic nanoantennas have also opened up an effective way of manipulating and directing light from localized photon sources and quantum emitters ${ }^{3,5,6}$ via near-field interactions providing directed emission of light from localized light sources. ${ }^{5,7-12}$ Furthermore, plasmonic nanoantennas can provide an efficient way to couple light from free space into plasmonic ${ }^{13-15}$ or dielectric $^{16,17}$ waveguides and vice versa. Thus, they can serve as a new type of waveguide coupler for optical chips.

Typically, directional farfield scattering by plasmonic nanoantennas is achieved by detuning the resonance wavelengths of two or more antenna elements and, thereby, adjusting their relative phase differences in the radiation fields. This results in constructive interference of radiation in one direction and destructive interference in the other direction. Since the sign of the relative phase difference between the antenna elements usually does not change with the wavelength of the incident light, most of the plasmonic nanoantennas investigated to date are designed for directional scattering of the incident light at one operation wavelength and into one direction only. $5,13-21$ However, in order to increase optical data-communication speed and bandwidth, it is essential to provide nanoantennas with multi-frequency operation ${ }^{22}$ that allows to increase information density by parallel encoding of information at different operation wavelengths (multiplexing). In such a scheme wavelength-selective bidirectional nanoantenna-waveguide coupling to separate information encoded in the wavelength is an essential device for on-chip light demultiplexing. To realize bidirectional nanoantennas, however, a strong relative phase difference with opposite signs has to be realized. One promising way of achieving this goal is the use of Fano resonances, ${ }^{23,24}$ where the constructive and destructive inference of two resonances with very different radiation characteristics

\footnotetext{
${ }^{\text {a) }}$ Author to whom correspondence should be addressed. Electronic mail: manuel.decker@anu.edu.au.
}

provide positive and negative relative radiation phases in a given wavelength range. Fano resonances in plasmonic nanostructures have already been found to support bidirectional radiation of light from localized quantum emitters, ${ }^{7,9,10}$ however, studies of bidirectional scattering for farfield excitation are, so far, limited to the directional excitation of surfaceplasmon polaritons ${ }^{13-15}$ or the use of the interband transitions of bi-metallic dimers ${ }^{25}$ which are all connected with large losses ${ }^{13,15,25}$ or are not scalable with regard to their operation frequency. ${ }^{25}$ Furthermore, a bidirectional nanoantenna design providing the functionality of a bidirectional waveguide coupler for wavelength demultiplexing has not been presented to date.

Here, we present a compact single-particle Fano nanoantenna that shows the capability of simultaneously directing light of two different wavelengths in opposite directions for broadband farfield excitation in free space [see Fig. 1(a)]. We demonstrate that this Fano antenna can be integrated onto a high-index dielectric waveguide and can be utilized as a bidirectional waveguide coupler for wavelength demultiplexing. In order to achieve bidirectionality, we effectively merge a rod-shaped aperture antenna and a circular patch antenna into one compact subwavelength nanoparticle. We utilize the interference of the radiation from those two elements that can be regarded as two electric dipoles with very different resonance widths in order to achieve wavelengthselective bidirectional scattering [Fig. 1(b)]. The first electric-dipole resonance with a broad linewidth is obtained from a gold nanodisk with a diameter of $d=400 \mathrm{~nm}$ and a thickness of $h=30 \mathrm{~nm}$. To realize the second electric-dipole resonance with approximately the same resonance frequency but a narrow linewidth (as required for the formation of a Fano resonance), we inscribe a 15-nm-wide and 120-nmlong nanoslit in $x$ direction [see Fig. 2(a)] and displace it by $s=100 \mathrm{~nm}$ from the center of the gold nanodisk. To intuitively understand how the Fano-antenna characteristics shown in Fig. 2(a) give rise to bidirectional scattering for different operation wavelengths, we describe the two elements of the Fano antenna (the nanodisk and the nanoslit) as two electric dipoles $\left(P_{a}\right.$ and $\left.P_{b}\right)$ oriented in $y$ direction, one positioned at the nanodisk center and the other at the center of the nanoslit [see inset Fig. 2(a)]. The radiation characteristics 

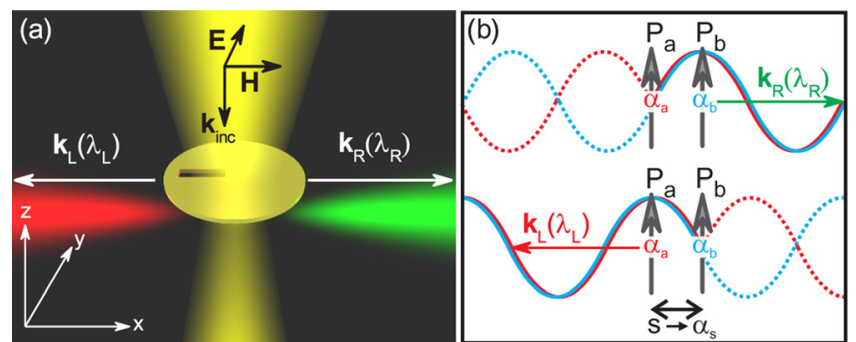

FIG. 1. (a) Artist view of the bidirectional Fano nanoantenna. The incident broadband light (depicted in yellow) is scattered in opposite directions $\left(\mathbf{k}_{L}\left(\lambda_{L}\right), \mathbf{k}_{R}\left(\lambda_{R}\right)\right)$ for different wavelengths $\left(\lambda_{L}\right.$ red and $\lambda_{R}$ green). (b) Dipole scheme showing the interference conditions for bidirectional scattering with $\mathbf{k}_{L}\left(\lambda_{L}\right)$ and $\mathbf{k}_{R}\left(\lambda_{R}\right)$.

are then governed by the response of the two (ideal) dipole sources with equal amplitudes $P_{a}=e^{i \alpha_{a}}$ and $P_{b}=e^{i \alpha_{b}}$, where $\alpha_{a}$ and $\alpha_{b}$ represent the phases of the respective dipole radiation [see Fig. 1(b)]. Furthermore, the relative displacement

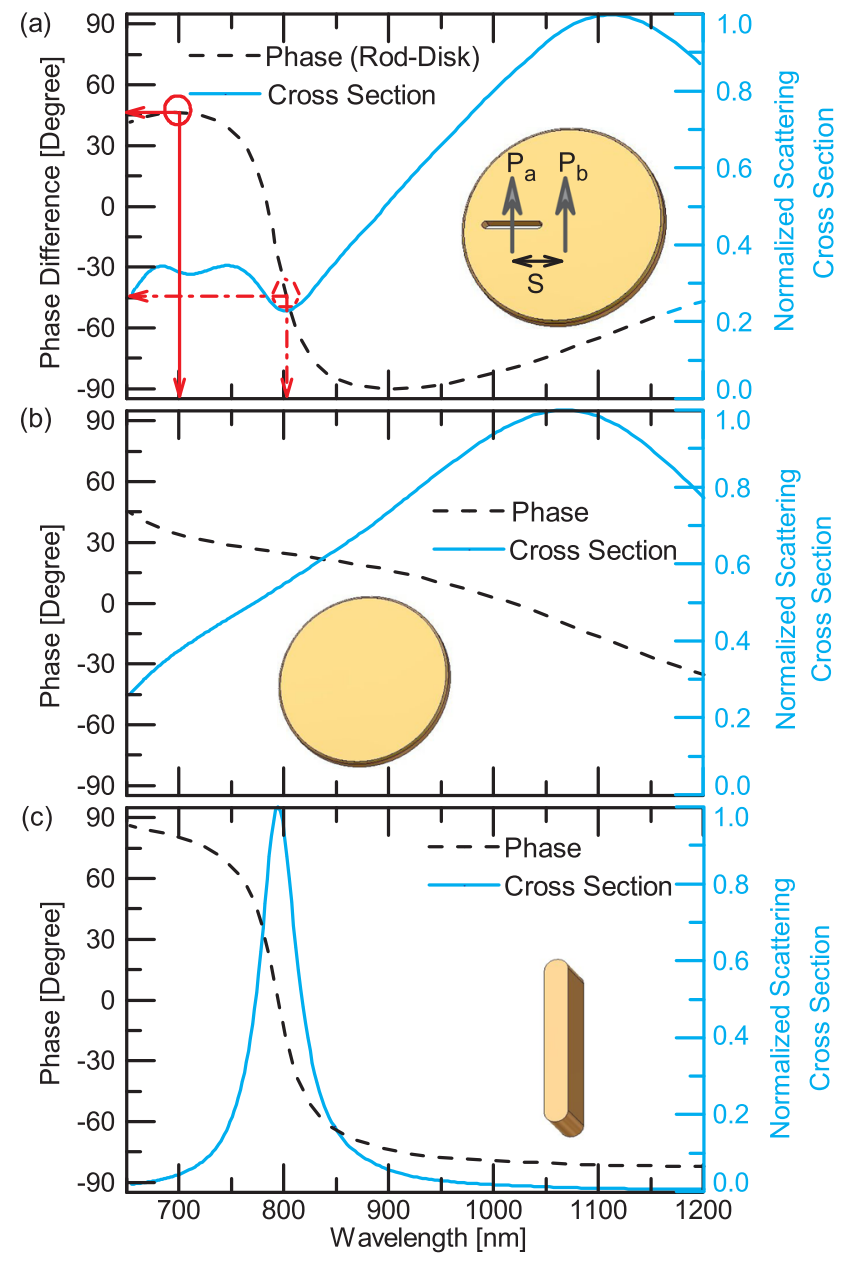

FIG. 2. (a) Normalized scattering cross section (blue curve) and the phase difference between the single nanorod and the nanodisk (black dashed curve). The inset shows the Fano antenna design. The two arrows indicate the dipoles $P_{a}$ and $P_{b}$ representing the nanoslit and the nanodisk, respectively. At $702 \mathrm{~nm}$ (phase difference of $+47^{\circ}$ ) and at $808 \mathrm{~nm}$ (phase difference of $-45^{\circ}$ ) bidirectional scattering occurs. (b) Normalized scattering cross section (blue curve) and phase spectrum (black dashed curve) of a single gold nanodisk with a diameter $400 \mathrm{~nm}$ and a thickness $30 \mathrm{~nm}$. (c) Normalized scattering cross section (blue curve) and phase spectrum (black dashed curve) of a gold nanorod with a length of $120 \mathrm{~nm}$, a width of $15 \mathrm{~nm}$, and $30 \mathrm{~nm}$ thickness. $s=100 \mathrm{~nm}$ of the two dipoles with respect to each other introduces a retardation phase factor $\alpha_{s}$. In order to achieve scattering to the left ( $-x$ direction), the conditions $\alpha_{L}=\left(\alpha_{a}-\alpha_{b}\right)+\alpha_{s}=0^{\circ}$ (constructive interference to the left) and $\alpha_{R}=-\left(\alpha_{a}-\alpha_{b}\right)+\alpha_{s}=180^{\circ}$ (destructive interference to the right) have to be fulfilled. Likewise, for scattering to the right ( $+x$ direction), $\alpha_{L}=\left(\alpha_{a}-\alpha_{b}\right)+\alpha_{s}=180^{\circ}$ (destructive interference to the left) and $\alpha_{R}=-\left(\alpha_{a}-\alpha_{b}\right)+\alpha_{s}=0^{\circ}$ (constructive interference to the right) have to be valid. While one of these two conditions, corresponding to unidirectional scattering only, can be met quite easily, fulfilling both conditions at the same time is impossible for the ideal case of bidirectional scattering with infinite front-to-back ratio (zero back scattering) since this implies that $\Delta \alpha=\alpha_{a}-\alpha_{b}= \pm 90^{\circ}$ and $\alpha_{s}=90^{\circ}$ have to be fulfilled simultaneously. However, if one allows non-zero backscattering $\left(|\Delta \alpha|=\alpha_{s}\right)$, bidirectional scattering with a maximized front-to-back ratio can be achieved.

In order to investigate the radiation properties of the actual Fano antenna shown in Fig. 2(a), we perform numerical calculations using finite-integral frequency-domain simulations (CST Microwave Studio). First, we excite the Fano antenna that is suspended in air with a linearly polarized plane wave incident from the top and calculate the normalized scattering cross section. Figure 2(a) shows that the scattering cross section is characterized by a broad resonance with a pronounced maximum at $1100 \mathrm{~nm}$ wavelength and a resonance minimum at $800 \mathrm{~nm}$ wavelength. To identify the origin of the Fano resonance in our nanoantenna design, we analyze the constituent elements of the antenna, namely, the gold nanodisk and the nanoslit, separately. For a single gold nanodisk [Fig. 2(b)] with a diameter of $d=400 \mathrm{~nm}$ and a thickness of $h=30 \mathrm{~nm}$, we find a broad resonance with a pronounced peak at about $1070 \mathrm{~nm}$ wavelength in the scattering cross section [solid line in Fig. 2(b)]. This peak can clearly be identified as the main resonance found for the Fano antenna shown in Fig. 2(a). To evaluate the phase of the scattered field, we position an electric-field probe in the nearfield of the nanodisk and extract the phase delay. Notably, the large resonance width of the nanodisk is connected with a weak phase dispersion.

Inspired by Babinet's principle, ${ }^{26}$ we investigate the scattering properties of the complementary structure - a single gold nanorod with the same dimensions [Fig. 2(c)], in order to analyze the optical response of the nanoslit. According to Babinet's principle, the radiation pattern of the nanoslit is identical in shape to that of a nanorod except that electric and magnetic fields are interchanged. ${ }^{26,27}$ In order to describe the response of the nanoslit that is excited by an incident electric field perpendicular to the slit by its complementary structure, a nanorod, the orientation of the nanorod has to be parallel to the polarization of the incident light. Thus, we calculate the response of a gold nanorod with the same dimensions as the nanoslit in the final nanoantenna design but with perpendicular orientation [see Fig. 2(c)]. Notably, since Babinet's principle is not exact for (lossy) optical metals, the nanoslit resonance is slightly blueshifted and spectrally broadened as compared to the nanorod resonance, however, these small deviations do only marginally affect the results obtained from the dipole model. We find that the nanorod shows a resonance 
(scattering peak) at $800 \mathrm{~nm}$ wavelength that can be linked to the resonance of the nanoslit. Furthermore, the nanorod resonance is relatively narrowband in comparison to the resonance of the nanodisk. This is connected with a strong phase dispersion in the scattered field at $800 \mathrm{~nm}$ wavelength. The interference of the radiation of the nanodisk and the nanoslit in the final nanoantenna design ultimately leads to a Fano resonance in the scattering cross section at $800 \mathrm{~nm}$ wavelength as can be seen in Fig. 2(a). Furthermore, the Fano resonance results in a relative phase difference $\Delta \alpha$ of the scattered field of the nanodisk and the complementary nanoslit that is positive for wavelengths smaller than approximately $790 \mathrm{~nm}$ and negative for wavelengths above this value [dashed line in Fig. 2(a)]. Specifically, we obtain $\Delta \alpha=\alpha_{a}-\alpha_{b}=-45^{\circ}$ at $808 \mathrm{~nm}$ wavelength and $\Delta \alpha=\alpha_{a}-\alpha_{b}=+47^{\circ}$ at $702 \mathrm{~nm}$ wavelength for our actual Fano antenna design. Because of the fixed displacement of the two dipoles $s=100 \mathrm{~nm}$ at $808 \mathrm{~nm}$ wavelength, the retardation phase is fixed to $\alpha_{s} \approx 45^{\circ}$. As a result, we expect our Fano antenna to support scattering to the right at $702 \mathrm{~nm}$ wavelength and to the left at $808 \mathrm{~nm}$ wavelength for excitation with a $y$ polarized wave propagating in $-z$ direction [Fig. 1(a)]. To verify the bidirectional scattering of our Fano antenna, we now calculate the radar cross sections $R C S_{R}$ in $+x$ direction and $R C S_{L}$ in $-x$ direction and plot the corresponding (black and red) curves in Fig. 3(a). Clearly at $702 \mathrm{~nm}$ wavelength, the right scattering cross section $\left(R C S_{R}\right)$ is maximized, while the left scattering $\left(R C S_{L}\right)$ is minimal and vice versa at $808 \mathrm{~nm}$ wavelength. Furthermore, the side scattering ratios $S S R_{R}=R C S_{R} / R C S_{L}$ and $S S R_{L}=R C S_{L} / R C S_{R}$ as depicted in Fig. 3(b) exceed 17 for both scattering directions. The calculated directivity of the Fano antenna in $+x$ (at $702 \mathrm{~nm}$ ) and $-x$ (at $808 \mathrm{~nm}$ ) direction is 2.2 and 2.7, respectively.

In the next step, we integrate the bidirectional Fano antenna onto a chalcogenide-glass waveguide to couple light with different wavelengths into opposite waveguide

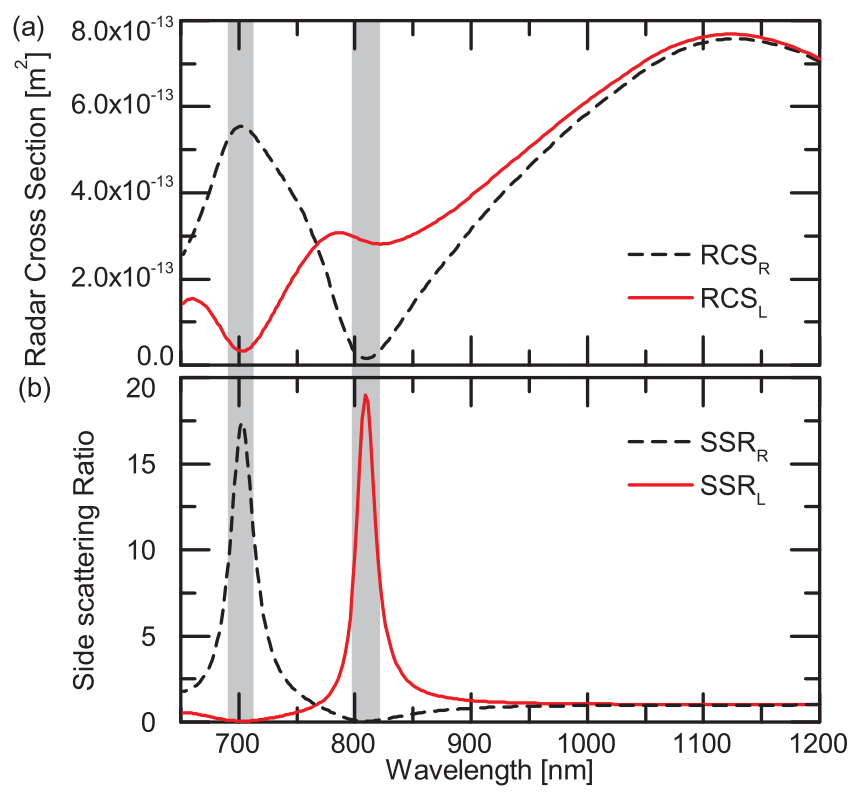

FIG. 3. (a) Radar cross section $R C S_{R}$ for scattering to the right ( $+x$ direction) and $R C S_{L}$ for scattering to the left ( $-x$ direction). (b) Side scattering ratios $S S R_{R}=R C S_{R} / R C S_{L}$ (black dashed curve) and $S S R_{L}=R C S_{L} / R C S_{R}$ (red solid curve). directions. We excite the antenna that is immersed in a droplet of index-matching liquid with the $y$-polarized mode of a tapered optical fibre (core diameter of $1 \mu \mathrm{m}$ ) from the top [see Figs. 4(a) and 4(b)]. The waveguide width is $600 \mathrm{~nm}$, height is $580 \mathrm{~nm}$, and the refractive index of the chalcogenide glass is 2.65 . The refractive index of the glass substrate, the index-matching liquid, and the glass fibre is 1.45. To quantify the amount of light coupled into the waveguide in $+x$ and $-x$ direction, we monitor the energy flow at the faces at each end of the waveguide. We then calculate the direction-dependent coupling efficiencies, i.e., the power flow through each of the faces normalized to the incident power flow from the fibre port. In doing so we obtain the spectrally resolved coupling efficiencies $\eta_{L}$ and $\eta_{R}$ for the left and the right wavguide face [Fig. 4(c)]. For the Fano antenna integrated onto a chalcogenide waveguide, we obtain maximum coupling efficiencies of $\approx 5 \%$ for each direction. This is quite impressive considering the subwavelength scale of both the nanoantennas and the waveguide.

Figure 4(d) finally shows the fidelity of the directional coupling, i.e., the ratios of the power coupled to the opposite waveguide directions for each wavelength. In our calculations, we obtain a maximum fidelity of 11 for coupling to the right direction at $1250 \mathrm{~nm}$ wavelength and a fidelity of 6.2 at $1610 \mathrm{~nm}$ for coupling to the left direction. At a wavelength
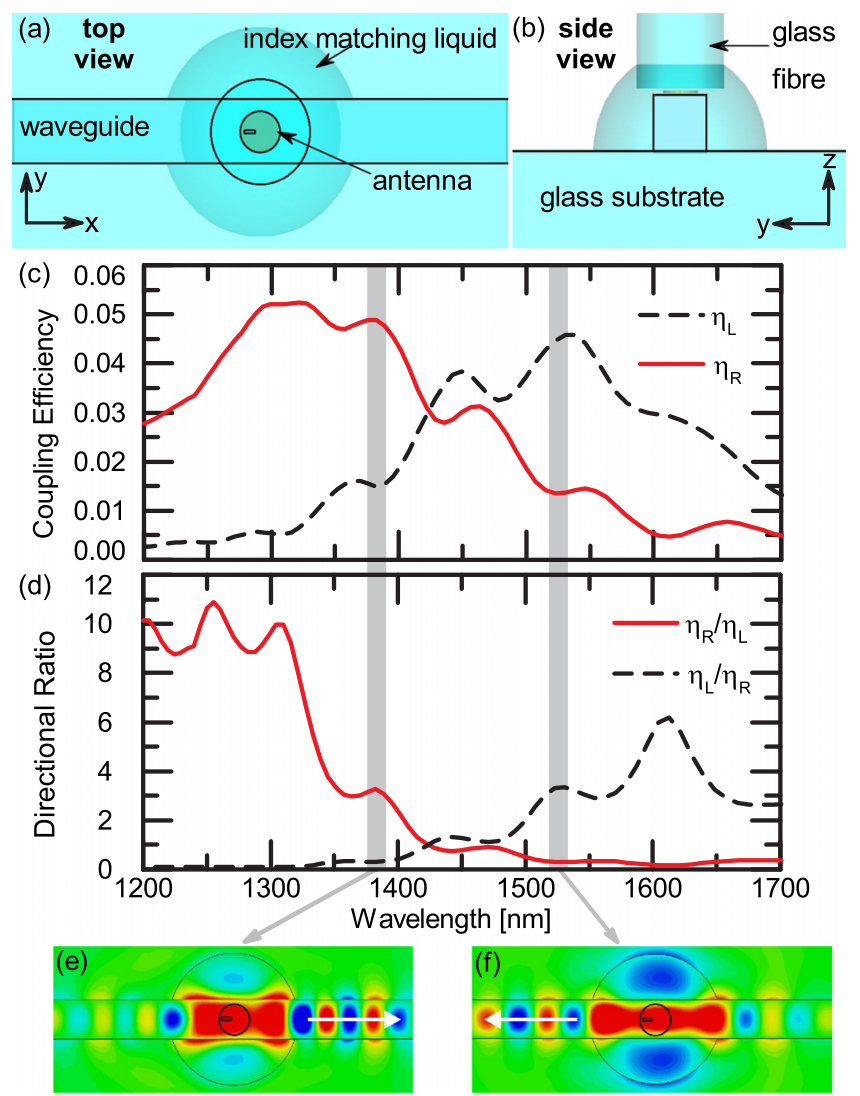

FIG. 4. (a) Top view and (b) side view of the Fano nanoantenna on a chalcogenide-glass waveguide. The antenna is excited by a glass fibre from the top. (c) Coupling efficiencies in $+x$ direction $\left(\eta_{R}\right.$, red solid curve) and in $-x$ direction ( $\eta_{L}$, black dashed curve) of the waveguide. (d) The directional ratio $\eta_{R} / \eta_{L}$ (red solid curve) and $\eta_{L} / \eta_{R}$ (black dashed curve) defines the fidelity of the directional coupling. (e) and (f) Top-view color plots of the $y$ component of the electric field in a cutting plane through the center of the waveguide. 
of around $1530 \mathrm{~nm}$ and $1380 \mathrm{~nm}$, we get a comparable fidelity of about 3.3 for both directions. For these two cases, we additionally plotted the $y$ component of the electric field in a cutting plane through the center of the waveguide. Figures 4(e) and 4(f) visualize the bidirectional coupling that is realized at around $1380 \mathrm{~nm}$ and $1530 \mathrm{~nm}$ wavelength showing clearly that, for the two selected wavelengths, the waveguide mode is launched mostly in one direction.

In summary, we have introduced a single-element nanoantenna consisting of a gold nanodisk and a Babinet-inverted nanorod (nanoslit). We have shown that this nanoantenna exhibits a pronounced Fano response that can be exploited to generate bidirectional radiation patterns for different wavelengths of light with a maximum side-scattering ratio of approximately 17 for opposite directions. We also demonstrate that this bidirectional Fano antenna can be integrated onto dielectric waveguides and can provide an on-chip solution for two-colour routing that may lead to compact devices for wavelength-selective waveguide demultiplexing. Finally, due to the Fano resonance, the antenna properties are inherently sensitive to changes in the dielectric environment which makes this Fano antenna a promising candidate for integrated sensing applications.

We thank W. Liu and A. Miroshnichenko for useful discussions.

${ }^{1}$ J. A. Schuller, E. S. Barnard, W. Cai, Y. C. Jun, J. S. White, and M. L. Brongersma, Nat. Mater. 9, 193 (2010).

${ }^{2}$ L. Novotny and N. F. van Hulst, Nat. Photonics 5, 83 (2011).

${ }^{3}$ V. Giannini, A. I. Fernández-Domínguez, S. C. Heck, and S. A. Maier, Chem. Rev. 111, 3888 (2011).

${ }^{4}$ P. Biagioni, J.-S. Huang, and B. Hecht, Rep. Prog. Phys. 75, 024402 (2012).

${ }^{5}$ A. G. Curto, G. Volpe, T. H. Taminiau, M. P. Kreuzer, R. Quidant, and N. F. van Hulst, Science 329, 930 (2010).

${ }^{6}$ M. Decker, I. Staude, I. I. Shishkin, K. B. Samusev, P. Parkinson, V. K. Sreenivasan, A. Minovich, A. E. Miroshnichenko, A. Zvyagin, C.
Jagadish, D. N. Neshev, and Y. S. Kivshar, Nat. Commun. 4, 2949 (2013).

${ }^{7}$ A. Artar, A. A. Yanik, and H. Altug, Nano Lett. 11, 3694 (2011).

${ }^{8}$ I. S. Maksymov, I. Staude, A. E. Miroshnichenko, and Y. S. Kivshar, Nanophotonics 1, 65 (2012).

${ }^{9}$ S. H. Alavi Lavasani and T. Pakizeh, J. Opt. Soc. Am. B 29, 1361 (2012).

${ }^{10}$ M. V. Rybin, P. V. Kapitanova, D. S. Filonov, A. P. Slobozhanyuk, P. A. Belov, Y. S. Kivshar, and M. F. Limonov, Phys. Rev. B 88, 205106 (2013).

${ }^{11}$ Z. Xi, Y. Lu, W. Yu, P. Yao, P. Wang, and H. Ming, Opt. Express 21, 29365 (2013).

${ }^{12}$ I. M. Hancu, A. G. Curto, M. Castro-López, M. Kuttge, and N. F. van Hulst, Nano Lett. 14, 166 (2014).

${ }^{13}$ J. S. Q. Liu, R. A. Pala, F. Afshinmanesh, W. Cai, and M. L. Brongersma, Nat. Commun. 2, 525 (2011).

${ }^{14}$ Y. Liu, S. Palomba, Y. Park, T. Zentgraf, X. Yin, and X. Zhang, Nano Lett. 12, 4853 (2012).

${ }^{15}$ J. Lin, J. P. B. Mueller, Q. Wang, G. Yuan, N. Antoniou, X.-C. Yuan, and F. Capasso, Science 340, 331 (2013).

${ }^{16}$ F. Bernal Arango, A. Kwadrin, and A. F. Koenderink, ACS Nano 6, 10156 (2012).

${ }^{17}$ T. P. H. Sidiropoulos, S. A. Maier, and R. F. Oulton, Opt. Express 20, 12359 (2012).

${ }^{18}$ D. Dregely, R. Taubert, J. Dorfmüller, R. Vogelgesang, K. Kern, and H. Giessen, Nat. Commun. 2, 267 (2011).

${ }^{19}$ W. Zhu, D. Wang, and K. B. Crozier, Nano Lett. 12, 6235 (2012).

${ }^{20}$ W. Liu, A. E. Miroshnichenko, D. N. Neshev, and Y. S. Kivshar, ACS Nano 6, 5489 (2012).

${ }^{21}$ D. Vercruysse, Y. Sonnefraud, N. Verellen, F. B. Fuchs, G. Di Martino, L. Lagae, V. V. Moshchalkov, S. A. Maier, and P. Van Dorpe, Nano Lett. 13, 3843 (2013).

${ }^{22}$ I. Staude, I. S. Maksymov, M. Decker, A. E. Miroshnichenko, D. N. Neshev, C. Jagadish, and Y. S. Kivshar, Phys. Status Solidi RRL 6, 466 (2012).

${ }^{23}$ B. Luk'yanchuk, N. I. Zheludev, S. A. Maier, N. J. Halas, P. Nordlander, H. Giessen, and C. T. Chong, Nat. Mater. 9, 707 (2010).

${ }^{24}$ A. E. Miroshnichenko, S. Flach, and Y. S. Kivshar, Rev. Mod. Phys. 82, 2257 (2010).

${ }^{25}$ T. Shegai, S. Chen, V. D. Miljković, G. Zengin, P. Johansson, and M. Käll, Nat. Commun. 2, 481 (2011).

${ }^{26}$ C. A. Balanis, Antenna Theory: Analysis and Design (John Wiley \& Sons, 2012).

${ }^{27}$ N. Feth, S. Linden, M. W. Klein, M. Decker, F. B. P. Niesler, Y. Zeng, W. Hoyer, J. Liu, S. W. Koch, J. V. Moloney, and M. Wegener, Opt. Lett. 33, 1975 (2008). 